\title{
APPROXIMATELY CONVEX AVERAGE SUMS OF UNBOUNDED SETS
}

\author{
M. ALI KHAN ${ }^{1}$
}

ABstract. In this note we show that the average sum of a large but finite number of unbounded and open sets is approximately convex if their "degree of nonconvexity" is bounded.

I. Introduction. In [4], Loeb proved the following theorem for the nonstandard space $* R^{n}$.

THEOREM 1. Let $T$ be an internal star-finite set and, for each $t \in T$, let $v(t)$ be a vector in $n$-space ${ }^{*} R^{n}$ with $n v(t) \simeq 0$. For each internal set $B \subseteq T$, let $S(B)=\sum_{t \in B} v(t)$. Then the following is true: Given internal sets $B \subseteq T$ and $C \subseteq T$ and given $\lambda \in{ }^{*} R$ with $\lambda \in{ }^{*}(0,1)$, there exists an internal set $D \subseteq T$ with $S(D) \simeq \lambda S(B)+(1-\lambda) S(C)$.

[4] should be referred to for notation and references to nonstandard analysis.

Using this theorem, Brown [2] has shown that the average sum of a "large" number of bounded sets is approximately convex. In economic applications the sets one is concerned with are typically productions sets or the set of all points preferred to a given commodity bundle. Sometimes the structure of the problem is such that requiring these sets to be bounded is not a severe restriction. An example is the work on the existence of competitive equilibrium where the initial resources furnish a bound to these sets. However, in other problems, asking these sets to be bounded is a rather severe restriction. Such a situation arises when one wants to prove the equivalence between two solution concepts, e.g. between the core and competitive equilibria.

In this note we show how a particular measure of nonconvexity can be used to remedy this difficulty. A useful corollary of this exercise is that the assumption of compactness of the individual sets is not needed for

Received by the editors May 15, 1973 and, in revised form, July 16, 1973.

AMS (MOS) subject classifications (1970). Primary 52A20, 26A98; Secondary 90A99.

Key words and phrases. Average sum, convex sets, unbounded, degree of nonconvexity.

${ }^{1}$ I am grateful to an anonymous referee for his helpful comments.

(c) American Mathematical Society 1974 
the validity of the version of the Shapley-Folkman theorem most often used in economics.

II. Infinitely large number of sets. Let $T$ be an internal set of positive integers where $|T|$, the number of elements in $T$, is $\omega$, some infinite natural number. That is, $T=(1,2, \cdots, \omega)$ and $\omega \in{ }^{*} N-N$. Let $G \in \mathscr{P}\left({ }^{*} R^{n}\right)^{\mathrm{T}}$ be an internal correspondence from $T$ into ${ }^{*} R^{n}$. Define $(1 / \omega) \sum_{t \in T} G(t)$ as the set of points which are average sums of all internal selections from $\prod_{i=1}^{\omega} G(t)$. Finally a set $B$ in ${ }^{*} R^{n}$ is said to be $S$-convex if for all $x, y \in B$ and any $\lambda \in *(0,1)$ there exists a $z \in B$ such that $z \simeq \lambda x+(1-\lambda) y$. Using Theorem 1, the following can be shown.

THEOREM 2. ${ }^{2}$ If $G$ is an internal correspondence such that $(\exists M \in N)$ $(\forall t \in T)\{\|x(t)\| \leqq M$ for all $x(t) \in G(t)\}$, then $(1 / \sqrt{ } \omega) \sum_{t \in T} G(t)$ is $S$-convex.

Proof. Let $x, y$ be in $(1 / \sqrt{ } \omega) \sum_{t \in T} G(t)$. Then there exist internal functions $x(t)$ and $y(t)$ such that $(1 / \sqrt{ } \omega) \sum_{t \in T} x(t)=x$ and $(1 / \sqrt{ } \omega) \sum_{t \in T} y(t)=y$. Let $S(B)=\left[\sum_{t \in B}(x(t) / \sqrt{ } \omega), \quad \sum_{t \in B}(y(t) / \sqrt{ } \omega)\right]$. Now $S(\varnothing)=[0,0]$ and $S(T)=[x, y]$. Thus the hypotheses of Loeb's theorem are satisfied and for any given $\lambda$ there exists an internal set $D \subseteq T$ such that $S(D) \simeq$ $[(1-\lambda) x,(1-\lambda) y]$. Therefore $S(T-D) \simeq[\lambda x, \lambda y]$. Define the function $h(t)$ as

$$
\begin{array}{rlrl}
h(t) & =x(t) \quad & (\forall t \in D), \\
& =y(t) \quad(\forall t \in T-D) .
\end{array}
$$

Since $x(t), y(t)$ are internal functions and $D, T-D$ internal sets, $h(t)$ is an internal function. Further, it can be easily seen that $(1 / \sqrt{ } \omega) \sum_{t \in T} h(t) \simeq$ $\lambda y+(1-\lambda) x$. Q.E.D.

REMARK. A variant of this theorem was first proved by Brown [2].

Let the radius of a set $S, \operatorname{rad}(S)$, be the radius of the smallest ball containing $S$ i.e., $\operatorname{rad}(S)=\operatorname{Inf}_{x \in S} r(x)$ where $r(x)=\{r \mid B(x, r) \supseteq S\}$. A set $B$ is said to be $Q$-convex if for all $x, y \in B$ and any $\lambda \in(0,1)$ there exists a $z \in B$ such that $z=\lambda x+(1-\lambda) y$. The $Q$-convex hull of $B, Q$-con $(B)$, is the set of all internal star-finite convex combinations of points in $B$. Note that if $B$ is internal, $Q$-con $(B)$ is internal.

RemarK. If $B$ is internal, $Q$-con $(B)$ is $Q$-convex.

THEOREM 3. ${ }^{3}$ Let $B$ be $S$-convex; then for any $y \in Q$-con $(B)$ there exists $z \in B$ such that $z \simeq y$.

\footnotetext{
${ }^{2}$ Note that we need only assume that, for all $t \in T, G(t)$ consists of finite vectors. It can be easily seen that, since $(G(t))_{t \in T}$ is an internal family of internal sets of finite vectors, it is uniformly bounded. I am indebted to the referee for this remark.

${ }^{3}$ Under a different wording this theorem was proved by Brown [2].
} 
Proof. If $B$ is $S$-convex, for any $x_{i} \in B$ and $\lambda_{i} \geqq 0, \sum_{i=1}^{v} \lambda_{i}=1, v \in{ }^{*} N$, there exists $z \in B$ such that $z \simeq \sum_{i=1}^{v} \lambda_{i} x_{i}$. We can prove this by induction. By the definition of $S$-convexity the statement is true for $v=2$. Let it hold for $v=n-1$. We may suppose that $0<\lambda_{n}<1$; otherwise there is nothing to prove. Let $x^{\prime}=\left(\sum_{i=1}^{n-1} \lambda_{i} x_{i}\right) /\left(1-\lambda_{n}\right)$. Since $\sum_{i=1}^{n=1}\left(\lambda_{i} /\left(1-\lambda_{n}\right)\right)=1$, there exists $r$ in $B$ such that $r \simeq x^{\prime}$. However $\lambda_{n} x_{n}+\left(1-\lambda_{n}\right) x^{\prime}=\sum_{i=1}^{n} \lambda_{i} x_{i} \simeq$ $\lambda_{n} x_{n}+\left(1-\lambda_{n}\right) r$. Thus by the definition of $S$-convexity there exists $z \in B$ such that $z \simeq\left[\lambda_{n} x_{n}+\left(1-\lambda_{n}\right) r\right] \simeq \sum_{i=1}^{n} \lambda_{i} x_{i}$.

Now any $y \in Q-\operatorname{con}(B)$ can be written as $\sum_{i=1}^{v} \lambda_{i} x_{i}, \sum_{i=1}^{v} \lambda_{i}=1, x_{i} \in B$. From the above there exists $z \in B$ such that $z \simeq \sum_{i=1}^{v} \lambda_{i} x_{i}=y$. Q.E.D.

We now consider a measure of nonconvexity, originally due to Starr [5], although he worked only with compact sets. Let $x \in Q$-con $(S)$ and let $\mathscr{L}(x, S)=\{A \mid A \subseteq S, x \in Q$-con $(A)\}$. Then the inner radius of $S, R(S)$, is $\operatorname{Sup}_{x \in Q \text {-con }(S)} \operatorname{Inf}_{A \in \mathscr{L}} \operatorname{rad}(A)$. Note that if a particular set $S$ is $Q$-convex, $R(S)=0$. Thus the inner radius measures the degree of nonconvexity of a set. We can now show that Theorem 2 can be extended to sets which may be unbounded but whose degree of nonconvexity is bounded.

THEOREM 4. Let $G$ be an internal correspondence such that

$$
(\exists M \in N)(\forall t \in T)[R(G(t)) \leqq M] .
$$

Then $(1 / \sqrt{ } \omega) \sum_{t \in T} G(t)$ is $S$-convex.

Proof. We will show that for any $y \in Q-\operatorname{con}\left[(1 / \sqrt{ } \omega) \sum_{t \in T} G(t)\right]$ there exists $x \in(1 / \sqrt{ } \omega) \sum_{t \in T} G(t)$ such that $y \simeq x$. Now it is true by transfer that the operators $Q$-con and $(1 / \sqrt{ } \omega) \sum_{t \in T}$ commute, (see [1, p. 387]). Thus there exists an internal function $y(t)$ such that $y(t) \in Q$-con $(G(t))$ and $(1 / \sqrt{ } \omega) \sum_{t \in T} y(t)=y$. Thus, for all $t$ in $T$, there exists an internal set $S(t) \subseteq G(t)$ such that $y(t) \in Q$-con $(S(t))$ and $\operatorname{rad}(S(t)) \leqq M$. Since $S(t)-y(t)$ are standardly bounded sets for all $t$ in $T$, an application of Theorem 2 gives us the fact that $(1 / \sqrt{ } \omega) \sum_{t \in T}(S(t)-y(t))$ is $S$-convex. Thus $\left[(1 / \sqrt{ } \omega) \sum_{t \in T} S(t)\right]-y$ is $S$-convex which implies that $(1 / \sqrt{ } \omega) \sum_{t \in T} S(t)$ is $S$-convex. Finally, by an application of Theorem 4, we can see that there exists a $z \in(1 / \sqrt{ } \omega) \sum_{t \in T} S(t)$ such that $z \simeq y$. Since $S(t) \subseteq G(t)(\forall t \in T)$, the theorem is proved. Q.E.D.

We can now say something about the average sum of sets.

THeOREM 5. Let $G$ satisfy the assumptions of Theorem 4 . Then for any $y \in Q-\operatorname{con}\left[(1 / \omega) \sum_{t \in T} G(t)\right]$ there exists $x \in(1 / \omega) \sum_{t \in T} G(t)$ such that $\omega^{1 / 2}\|x-y\| \simeq 0$.

Proof. We can easily see that for any $S$-convex set $B, x \in Q-\operatorname{con}(B / \sqrt{ } \omega)$ implies that there exists $y \in B / \sqrt{ } \omega$ such that $\omega^{1 / 2}\|x-y\| \simeq 0$. Suppose not, i.e. for all $y \in B / \sqrt{ } \omega, \omega^{1 / 2}\|x-y\| \neq 0$. Multiplying throughout by 
$\sqrt{ } \omega$ gives us a contradiction to the fact that $B$ is $S$-convex. Applying Theorem 4 proves the theorem. Q.E.D.

III. Large but finite number of sets. In [1], the following theorem due to Shapley-Folkman and Starr is proved.

THEOREM 6. Let $F$ be a family (not necessarily finite) of compact sets $S$ such that, for some number $M, R(S) \leqq M$ for all $S \in F$. Then for any finite subfamily $F^{\prime} \subset F$ and any $x \in \operatorname{con} \sum_{S_{G \in} F^{\prime}} S$, there is a $y \in \sum_{S_{\in} F^{\prime}} S$ such that $\|x-y\|<M \sqrt{ } n$, where $n$ is the dimensionality of the space.

We can prove the following theorem.

THEOREM 7. Let $\{G(t)\}_{t \in N}$ be a sequence of nonempty subsets of $R^{n}$ having uniformly bounded inner radii, say less than $M$. Then

$$
\begin{aligned}
(\forall \varepsilon>0)(\exists m \in N)(\forall n \in N) & \left(\forall x \in \operatorname{con} \frac{1}{n} \sum_{t=1}^{n} G(t)\right) \\
& \times\left(\exists y \in \frac{1}{n} \sum_{t=1}^{n} G(t)\right)[n \geqq m \Rightarrow\|x-y\|<\varepsilon / \sqrt{ } n] .
\end{aligned}
$$

Proof. Suppose the theorem is false; then

$$
\begin{aligned}
(\exists \varepsilon>0)(\forall m \in N)(\exists n \in N) & \left(\exists x \in \operatorname{con} \frac{1}{n} \sum_{t=1}^{n} G(t)\right) \\
& \times\left(\forall y \in \frac{1}{n} \sum_{t=1}^{n} G(t)\right)[n \geqq m \Rightarrow\|x-y\| \geqq \varepsilon / \sqrt{ } n] .
\end{aligned}
$$

Hence by transfer the following sentence is a true statement about ${ }^{*} U$, the nonstandard universe:

$$
\begin{aligned}
(\exists \varepsilon>0)\left(\forall m \in{ }^{*} N\right)\left(\exists n \in{ }^{*} N\right)\left(\exists x \in \operatorname{con} \frac{1}{n} \sum_{t=1}^{n} G(t)\right) \\
\quad \times\left(\forall y \in \frac{1}{n} \sum_{t=1}^{n} G(t)\right)[n \geqq m \Rightarrow\|x-y\| \geqq \varepsilon / \sqrt{ } n] .
\end{aligned}
$$

Choose $v \in{ }^{*} N-N$, then there exists $\omega \in{ }^{*} N-N$, and

$$
x \in Q-\operatorname{con}(1 / \omega) \sum_{t=1}^{\omega} G(t)
$$

such that for all $y$ in $(1 / \omega) \sum_{t=1}^{\omega} G(t),\|x-y\| \geqq \varepsilon / \sqrt{ } \omega$. This is a contradiction to Theorem 5. Q.E.D.

REMARK. Note that Theorem 7 is a slightly different version of Theorem 6. However it is in the former spirit that the Shapley-Folkman 
theorem is used in economics. We quote ${ }^{4}$ from Arrow-Hahn's analysis of the relationship between the core and the competitive equilibria, "(the) conclusion has little force for small economies. But if both sides are divided by the number of members of the economy $E, \cdots$ the average discrepancy $\cdot$.. approaches zero for large economies."

Compactness of the sets, however, can be weakened even in the case of Theorem 6. All we need to assume is that the sets $S$ are closed and their asymptotic cones are positively semi-independent. The latter requirement guarantees that a finite sum of the sets $S$ is closed, see Debreu [3, p. 23]. This, in turn, shows that the proof of Arrow-Hahn [1, p. 399], need not be changed.

\section{REFERENCES}

1. K. J. Arrow and F. H. Hahn, General competitive analysis, Holden-Day, San Francisco, Calif., 1971.

2. D. J. Brown, Convexity of the vector average (mimeo).

3. G. Debreu, Theory of value: an axiomatic analysis of economic equilibrium, Cowles Foundation for Research in Economics at Yale University, Monograph 17, Wiley, New York; Chapman \& Hall, London, 1959. MR 22 \#1447.

4. P. A. Loeb, A combinatorial analog of Lyapunov's theorem for infinitesimally generated atomic vector measures, Proc. Amer. Math. Soc. 39 (1973), 585-586.

5. R. M. Starr, Quasi-equilibria in markets with nonconvex preferences, Econometrica, 37 (1969), 25-38.

1061 A Donnington Circle, Towson, Maryland 21204

Current address: Department of Political Economy, The Johns Hopkins University, Baltimore, Maryland 21218

\footnotetext{
${ }^{4}$ See [1, p. 191].
} 